\title{
HALLAZGOS MICROSCÓPICOS DEL TEJIDO PULMONAR Y LOS MÚSCULOS RESPIRATORIOS EN LA ENFERMEDAD PULMONAR OBSTRUCTIVA CRÓNICA (EPOC)
}

\author{
MICROSCOPIC FINDINGS OF LUNG TISSUE AND RESPIRATORY MUSCLES IN CHRONIC OBSTRUCTIVE \\ PULMONARY DISEASE (COPD)
}

Tales Lyra de Oliveira ${ }^{1,2, a}$, Tácio Nertan Cartaxo de Souza ${ }^{3, b}$, Byron Martins-Lucena,c, Fernanda Mayara Santos-Santana ${ }^{5, d}$, Lysien Ivania-Zambrano,e, , Ana Rose de Carvalho-Araujo ${ }^{7, f}$, Ana Lúcia de Gusmão-Freire ${ }^{8,9}$

\begin{abstract}
RESUMEN
Objetivo: Evaluar los cambios histopatológicos presentes en los pulmones, diafragma y esternocleidomastoideo en cadáveres con diagnóstico de Enfermedad Pulmonar Obstructiva Crónica. Métodos: Este es un estudio ciego y descriptivo con análisis de imágenes histológicas de biopsia. El historial de tabaquismo asociado con la presencia de antracosis pulmonar, engrosamiento septal y bullas enfisematosas comprendió los criterios de inclusión del estudio. La muestra consistió en biopsias de pulmón, diafragma y esternocleidomastoideo. El estudio se realizó mediante análisis microscópico óptico de láminas histológicas obtenidas de 36 cadáveres con Enfermedad Pulmonar Obstructiva Crónica. El diagnóstico histopatológico fue realizado por un patólogo que desconocía los objetivos del estudio. Resultados: En el diafragma, hubo la presencia de tejido adiposo interpuesto, atrofia muscular, eliminación de las fibras musculares y fibrosis. En el esternocleidomastoideo, también se evidenció la eliminación de las fibras musculares, la atrofia muscular, el tejido adiposo interpuesto, la hipertrofia muscular y el engrosamiento de los tendones. Conclusión: Los cambios encontrados en las biopsias musculares de diafragma y esternocleidomastoideo de los cadáveres con Enfermedad Pulmonar Obstructiva Crónica se evidenciaron como un mecanismo de compensación y/o disfunción del sistema respiratorio debido a alteraciones biomecánicas promovidas por la enfermedad.
\end{abstract}

Palabras clave: Enfermedad pulmonar obstructiva crónica; Músculo estriado; Antracosis; Tabaquismo; Autopsia (fuente: DeCS BIREME).

\begin{abstract}
Objective: To evaluate the histopathological changes present in the lungs and respiratory muscles (diaphragm and ECOM) of the corpses with a diagnosis of COPD. Methods: This is a blind and descriptive study with analysis of histological images of biopsy. The history of smoking associated with the presence of pulmonary anthracosis, septal thickening and emphysematous bullae included the inclusion criteria of the study. The sample consisted of lung, diaphragm and ECOM biopsies. The study was performed by optical microscopic analysis of histological sheets obtained from 36 bodies with COPD. The histopathological diagnosis was made by a pathologist who did not know the objectives of the study. Results: In the diaphragm, there was the presence of interposed adipose tissue, muscular atrophy, removal of muscle fibers and fibrosis. In ECOM, the elimination of muscle fibers, muscular atrophy, interposed adipose tissue, muscle hypertrophy and thickening of the tendons were also evident. Conclusion: The changes found in the diaphragm and ECOM muscle biopsies of the bodies with COPD were evidenced as a mechanism of compensation and / or dysfunction of the respiratory system due to biomechanical alterations promoted by the disease.
\end{abstract}

Key words: Chronic obstructive pulmonary disease; Striated muscle; Anthracosis; Tobacco Use Disorder; Autopsy (source: MeSH NLM).

\footnotetext{
${ }^{1}$ Facultad de Medicina, Universidad Municipal de São Caetano del Sur, São Paulo-Brasil.

2 Unidad de Hipertensión, Instituto del Corazón, Facultad de Medicina, Universidad de Sao Paulo, São Paulo-Brasil.

${ }^{3}$ Instituto de Ciencias Biológicas y de la Salud, Universidad Federal de Alagoas, Maceió-Brasil.

${ }^{4}$ Municipalidad de Maceió, Maceió-Brasil.

${ }^{5}$ Facultad de Medicina, Universidad Federal de Alagoas, Maceió-Brasil.

${ }^{6}$ Departamento de Ciencias Morfológicas, Facultad de Ciencias Médicas. Universidad Nacional Autónoma de Honduras, Tegucigalpa-Honduras.

7 Servicio de Verificación de Defunciones, Universidad Estatal de Ciencias de la Salud de Alagoas, Maceió-Brasil.

${ }^{8}$ Facultad de Fisioterapia, Universidad Estatal de Ciencias de la Salud de Alagoas, Maceió-Brasil.

a Fisioterapeuta, Doctor en Ciencias; ${ }^{b}$ Fisioterapeuta, Magíster en Ciencias de la Salud; ${ }^{C}$ Fisioterapeuta; ${ }^{\text {d }}$ Fisioterapeuta, Estudiante de Medicina;

e Médico Cirujano, Magíster en Ciencias; ${ }^{\ddagger}$ Médico Patólogo; 9 Fisioterapeuta, Magíster en Fisioterapia.

Citar como: Tales Lyra de Oliveira, Tácio Nertan Cartaxo de Souza, Byron Martins-Lucena, Fernanda Mayara Santos-Santana, Lysien IvaniaZambrano, Ana Rose de Carvalho-Araujo, Ana Lúcia de Gusmão-Freire. Hallazgos microscópicos del tejido pulmonar y los músculos respiratorios en la enfermedad pulmonar obstructiva crónica (EPOC) Rev. Fac. Med. Hum. Enero 2020; 20(1):55-63. DOI 10.25176/RFMH.v20i1.2447 


\section{INTRODUCCIÓN}

Más de 3 millones de personas murieron de Enfermedad Pulmonar Obstructiva Crónica (EPOC) en 2012, lo que representa el $6 \%$ de todas las muertes en todo el mundo ${ }^{(1-2)}$. En Brasil, la EPOC se considera la cuarta causa de muerte, afecta a más de 6 millones de personas y victimiza a aproximadamente 30000 personas por año(3). Los datos indican que $2 / 3$ de todas las autopsias en hombres y $1 / 7$ en mujeres muestran signos de enfisema pulmonar ${ }^{(4)}$. El estudio PLATINO, realizado en cinco regiones metropolitanas de América Latina, mostró que la prevalencia de EPOC en la población mayor de 40 años en São Paulo fue de $15.8 \%^{(5)}$.

La limitación crónica del flujo aéreo, característica de la EPOC, está causada predominantemente por una asociación de enfermedades de las vías respiratorias pequeñas (bronquiolitis obstructiva) y la destrucción del parénquima pulmonar (enfisema pulmonar), que promueve una afección de hiperinflación pulmonar ${ }^{(6)}$.

Ante la exposición crónica a patógenos, incluido el humo del cigarrillo, se produce una inflamación crónica en el parénquima pulmonar, lo que provoca un desequilibrio entre las enzimas proteolíticas $y$ antiprotolíticas ${ }^{(7)}$. Por lo tanto, cualquier factor que aumente la producción o actividad de las proteasas, especialmente las elastasas, y/o reduzca o incluso inhiba las anti-elastasas, promueve la destrucción de la estructura elástica pulmonar. La anti-elastasa más importante es la alfa-1-antitripsina, y la principal fuente de elastasa son los neutrófilos, que se elevan junto a los macrófagos, linfocitos $T$ y eosinófilos en los fumadores ${ }^{(8)}$. La alfa-1-antitripsina, que actúa para defender el tejido pulmonar contra las elastasas, está inactiva debido a la acción de las sustancias oxidantes liberadas por el humo del cigarrillo9. Por lo tanto, el enfisema pulmonar es el resultado del efecto elastolítico destructivo en fumadores con baja actividad anti-elastasa. Como la elastasa se expresa casi exclusivamente por los neutrófilos, los fumadores tienen más neutrófilos en su lavado bronco alveolar (BAL) que los no fumadores, por lo que los neutrófilos se consideran los mayores culpables de causar enfisema pulmonar ${ }^{(7)}$.

A partir de estos datos, es probable que exista una respuesta inflamatoria compleja en el pulmón enfisematoso. No se sabe exactamente cómo los diversos tipos de células inflamatorias contribuyen a la destrucción de las paredes alveolares, pero la especulación razonable es que los neutrófilos, macrófagos y eosinófilos degradan directamente la matriz extracelular alveolar mediante la liberación de proteinasas, mientras que los linfocitos $T$ influyen en el reclutamiento celular. Proteínas inflamatorias que liberan proteinasas ${ }^{(10)}$.

En la EPOC, el diafragma actúa contra el aumento de las cargas mecánicas debido a la limitación del flujo de aire y los cambios en la conformación del tórax debido a la condición de hiperinflación pulmonar. Se sugiere que esta sobrecarga mecánica impuesta en el diafragma simula el entrenamiento de resistencia ${ }^{(11)}$. La fuerza y la resistencia caracterizan el rendimiento muscular del diafragma. Por lo tanto, la pérdida de estas características da como resultado debilidad muscular diafragmática $y$, en consecuencia, su peor rendimiento mecánico ${ }^{(12)}$. La literatura ha demostrado sistemáticamente que las personas con EPOC grave generan menos presión inspiratoria máxima y presión transdiafragmática en maniobras voluntarias en comparación con las personas sin la enfermedad ${ }^{(13,14)}$. Por lo tanto, la fuerza muscular se considera un factor limitante en el desempeño del diafragma en pacientes con EPOC. Los músculos escaleno y esternocleidomastoideo (ECOM) son músculos inspiratorios con acciones similares en la pared torácica, que generan un desplazamiento craneal del esternón y las costillas. Sin embargo, estos músculos tienen diferentes patrones de actividad. Los escalenos son músculos inspiratorios activados en cada ciclo ventilatorio, incluso durante el reposo, mientras que los ECOM son músculos accesorios de inspiración y no se contraen durante la respiración basal, sino que lo hacen solo después de alcanzar aproximadamente el $70 \%$ de la capacidad inspiratoria(15).

A partir de estos datos, el presente estudio tuvo como objetivo evaluar los cambios histopatológicos presentes en los pulmones y los músculos respiratorios (diafragma y ECOM) de los cadáveres afectados por la EPOC.

\section{MÉTODOS}

\section{Diseño del estudio}

Este es un estudio descriptivo con hallazgos de imágenes de biopsia histológica utilizando una muestra no probabilística. La recolección de muestras se realizó en el Servicio de Verificación de la Muerte (SVO) de la Universidad Estatal de Ciencias de la Salud de Alagoas (UNCISAL), de abril a septiembre de 2012. El Comité de Ética de UNCISAL (CEP) aprobó el Protocolo 1796/2011 y los responsables legales de los cadáveres autopsiados fueron informados de los procedimientos y firmaron el formulario de consentimiento informado (CF) adjunto al protocolo general de admisión al SVO. Inicialmente, la información sobreel cadáver se recopiló 
del formulario de obituario de SVO, completada por el tutor legal, en la que se consideraron el género, la edad, la raza, la profesión, el origen y la presencia de antecedentes de tabaquismo, considerados los principales criterios de inclusión en el estudio.

Treinta y seis (36) cadáveres con diagnóstico histopatológico de EPOC confirmado por la presencia de antracosis pulmonar, engrosamiento septal y bullas enfisematosas se incluyeron en el estudio. Se excluyeron del estudio los cadáveres sin lóbulo o pulmón resultantes de cirugía ablativa o malformación congénita, con una presencia significativa de adherencias pleurales que sufrieron lesiones parenquimatosas por el procedimiento de necropsia y cadáveres con comorbilidades como enfermedad neuromuscular y malformación ósea estructural de la caja torácica. Así, se excluyeron del estudio ocho cadáveres, tres porque no presentaron los hallazgos histopatológicos esperados para la EPOC; cuatro por presentar adherencias pleurales intensas y uno por ser diagnosticado con enfermedad neuromuscular (enfermedad de Parkinson).

\section{Biopsias pulmonares y musculares}

El material para el análisis se recolectó mediante el procedimiento de necropsia estándar, que realizó una sección longitudinal desde la región de manubrio hasta la cicatriz umbilical para acceder a las estructuras torácicas y abdominales de las cuales se recolectaron muestras bilaterales de los ápices pulmonares (Figura 1.A). Las secciones de ECOM se realizaron bilateralmente en el lado ventral cerca de su inserción en el manubrio del esternón (Figura 1. B). Mientras que el músculo del diafragma se seccionó en su región lateral-costal derecha (Figura 1. C).
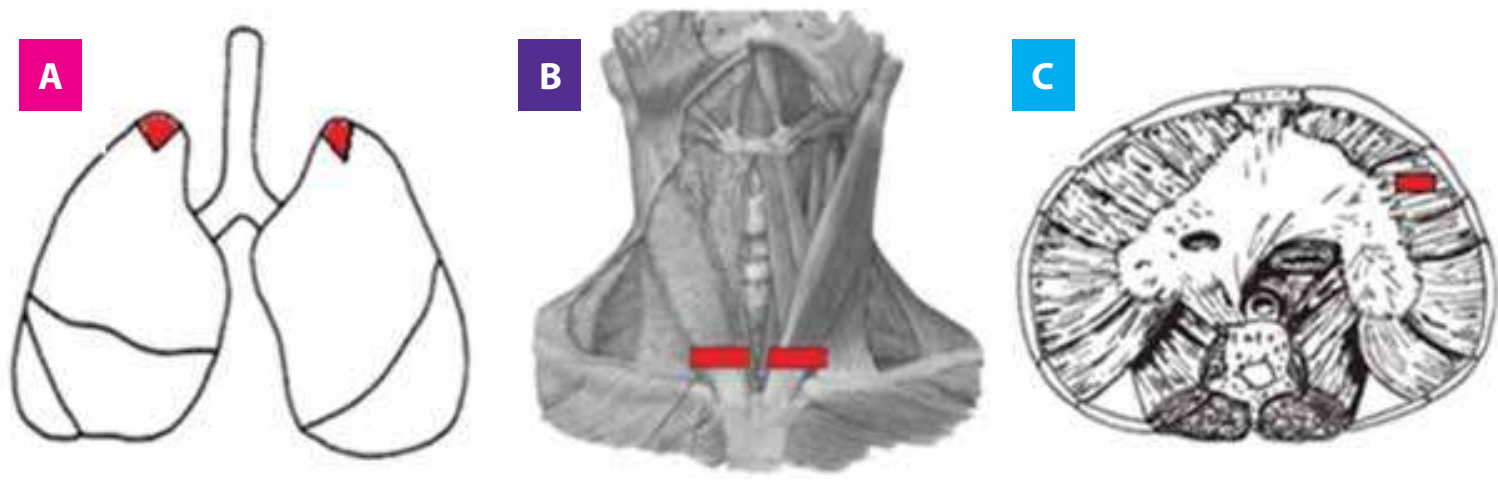

Figura 1. Secciones para el protocolo experimental. A) Sección en ápices pulmonares. B) Sección en el vientre muscular del ECOM, bilateralmente, cerca de su inserción en el manubrio; C) Sección en la región lateral-costal derecha del diafragma.

\section{Procesamiento histológico}

Se obtuvieron 5 fragmentos de tejido de cada cadáver para hacer un total de 288 láminas histológicas, lo que permitió la identificación y localización de cambios histopatológicos, por medio de dos tinciones diferentes. Para la visualización histológica, los tejidos se fijaron con formaldehído diluido al $10 \%$, se deshidrataron con diferentes concentraciones de etanol, se clarificaron con xilol y se incluyeron en parafina. Luego, el material de parafina se cortó a $5 \mu \mathrm{m}$ enespesordemicrótomoylassecciones desparafinadas se rehidrataron y se sometieron a tinción con hematoxilina-eosina $(\mathrm{HE})$ para la tinción estructural de todos los tejidos evaluados y la tinción tricrómica de Masson para fibrosis en tejidos musculares. Por lo tanto, fue necesario elaborar dos lâminas histológicas para cada tejido muscular, usando para una muestra la coloración por el método HE y el otro por el tricrómico de Masson. El parénquima pulmonar se evaluó solo mediante tinción con $\mathrm{HE}$. Las láminas histológicas se analizaron mediante microscopía óptica convencional y el diagnóstico histopatológico de las biopsias fue realizado por un patólogo de SVO, que desconocía los objetivos del estudio.

\section{RESULTADOS}

El perfil sociodemográfico de los cadáveres mostró que predominaban el género masculino $(63,9 \%, n=23)$, la raza mulata $(66,7 \%, \mathrm{n}=24)$, la profesión relacionada con el área urbana $(77,8 \%, n=28)$, la residencia en la capital $(61,1 \%, n=22)$ y la causa de muerte relacionada con enfermedades cardiopulmonares $(80,6 \%, n=29$, Tabla 1). El tiempo medio de tabaquismo de la muestra fue $31,3 \pm 19,6$ años. 
Tabla 1. Perfil socio demográfico de la muestra biopsiada de cadáveres con EPOC.

\section{Biopsias $(n=36)$}

Genero

Femenino

Masculino

$63,9 \%(23)$

Grupo de edad (años)

$67,2 \pm 17,4$

Raza

Branco

$30,5 \%(11)$

Mulato

$66,7 \%(24)$

Negro

$2,8 \%(1)$

Profesión

Zona Rural

$22,2 \%(8)$

Zona Urbana

$77,8 \%(28)$

Procedencia

Capital

Interior

$38,9 \%(14)$

Tiempo de tabaquismo (años)

$31,3 \pm 19,6$

Causa de muerte

Enfermedades Cardiopulmonares

$80,6 \%(29)$

Otras enfermedades

$19,4 \%(7)$

Los resultados de las biopsias de pulmón mostraron que la antracosis (pulmón D: $100 \%, n=36$; pulmón I: $94,4 \%, n=34$ ), las ampollas enfisematosas (pulmón $D$ y l: $66,7 \%, n=24$ ) y el engrosamiento septal (pulmón

D: $72,2 \%, n=26$; pulmón I: $77,8 \%, n=28$ ) fueron los hallazgos histopatológicos que estaban expresamente presentes en las muestras (Figura 2. A, B, C y D, respectivamente).
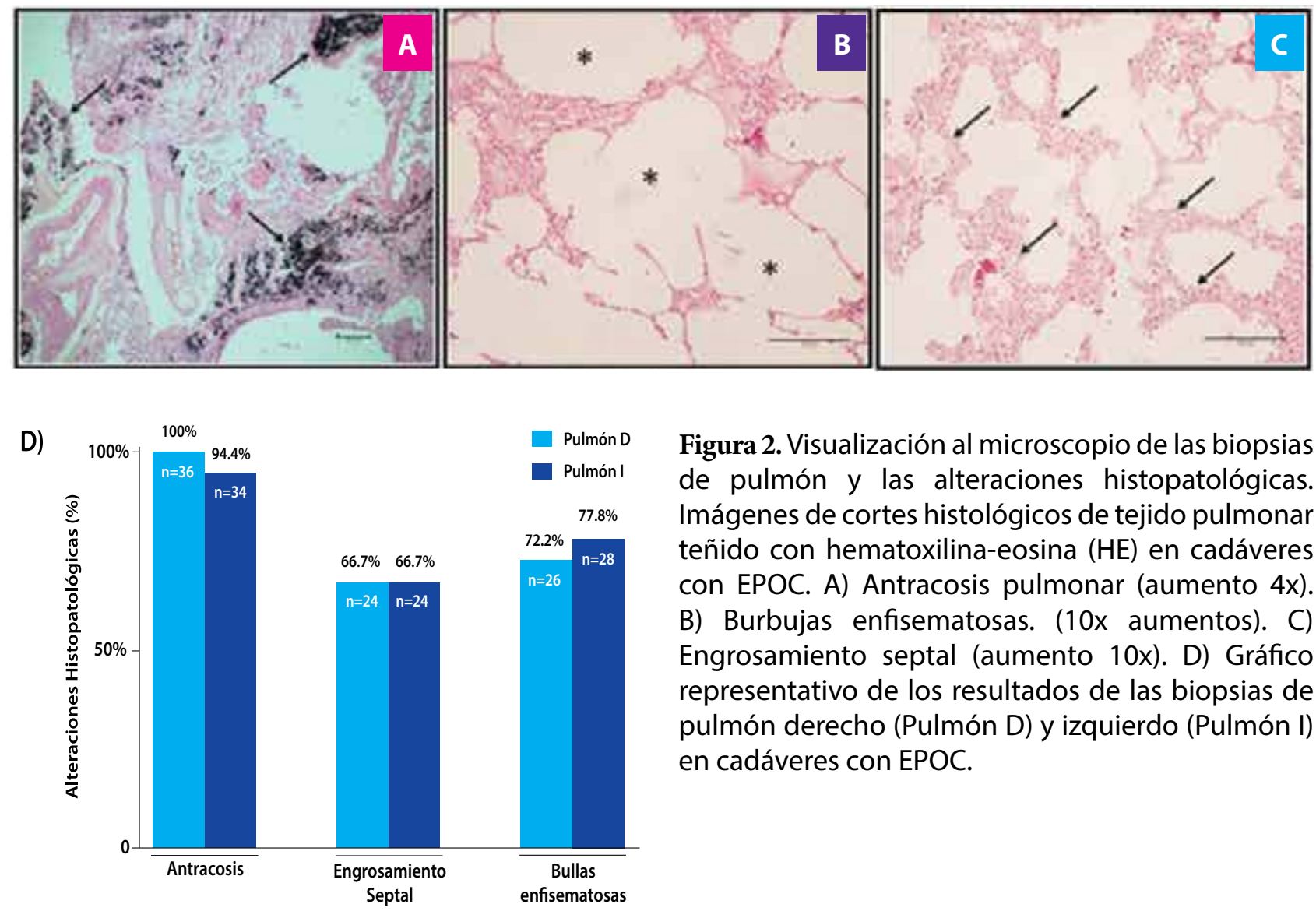

Figura 2. Visualización al microscopio de las biopsias de pulmón y las alteraciones histopatológicas. Imágenes de cortes histológicos de tejido pulmonar teñido con hematoxilina-eosina (HE) en cadáveres con EPOC. A) Antracosis pulmonar (aumento 4x). B) Burbujas enfisematosas. (10x aumentos). C) Engrosamiento septal (aumento 10x). D) Gráfico representativo de los resultados de las biopsias de pulmón derecho (Pulmón D) y izquierdo (Pulmón I) en cadáveres con EPOC. 
Con respecto a las biopsias de diafragma, los hallazgos más relevantes fueron la presencia de tejido adiposo interpuesto $(33,3 \%, n=12)$ y atrofia muscular $(25 \%$, $\mathrm{n}=9$ ), y en menor medida, el distanciamiento de las fibras musculares $(19,4 \%, n=7)$ y la instalación de fibrosis $(2,8 \%, n=1)$. Los resultados de las biopsias de diafragma se pueden ver en la Figura 3. A, B, C, D y E, respectivamente.
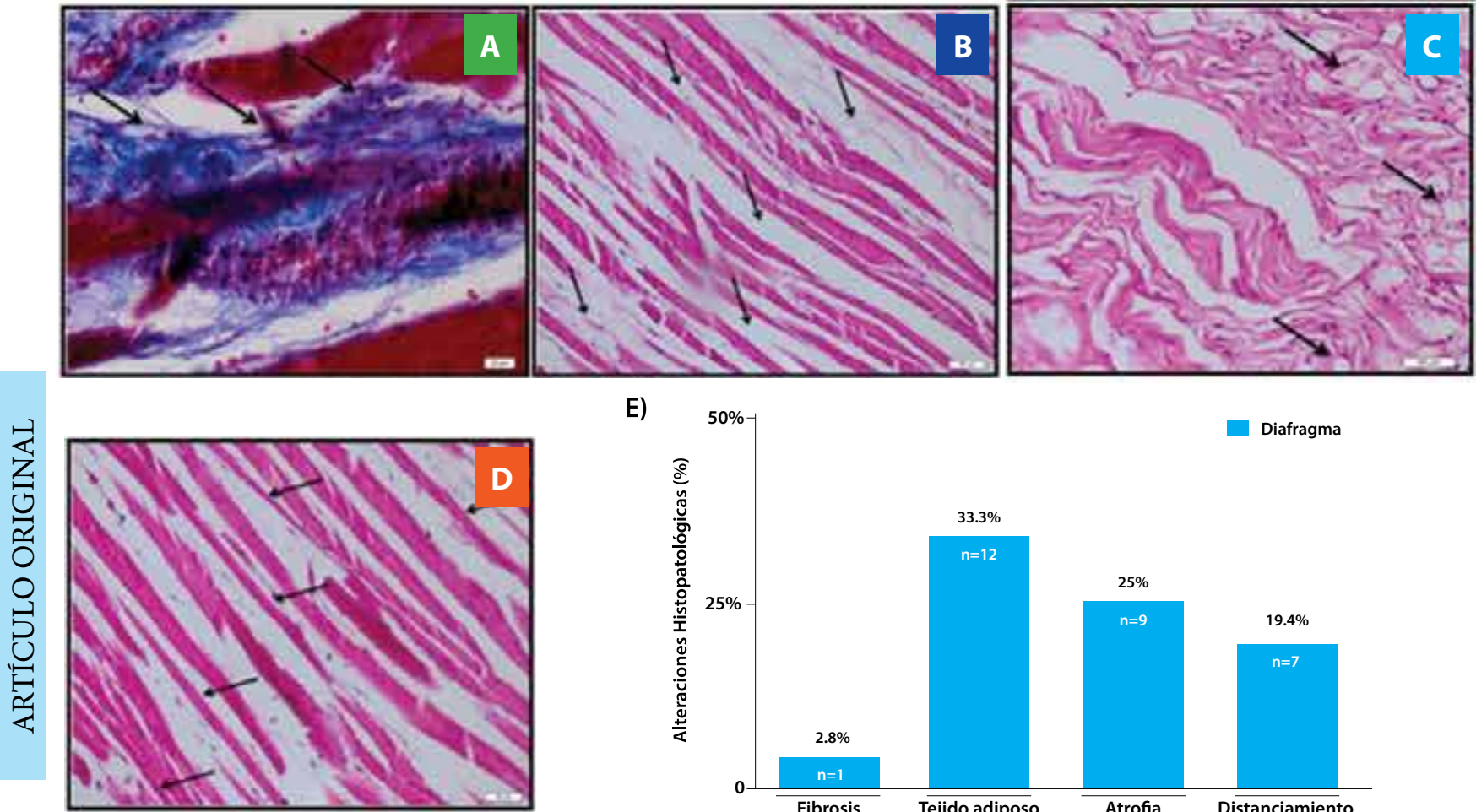

E)

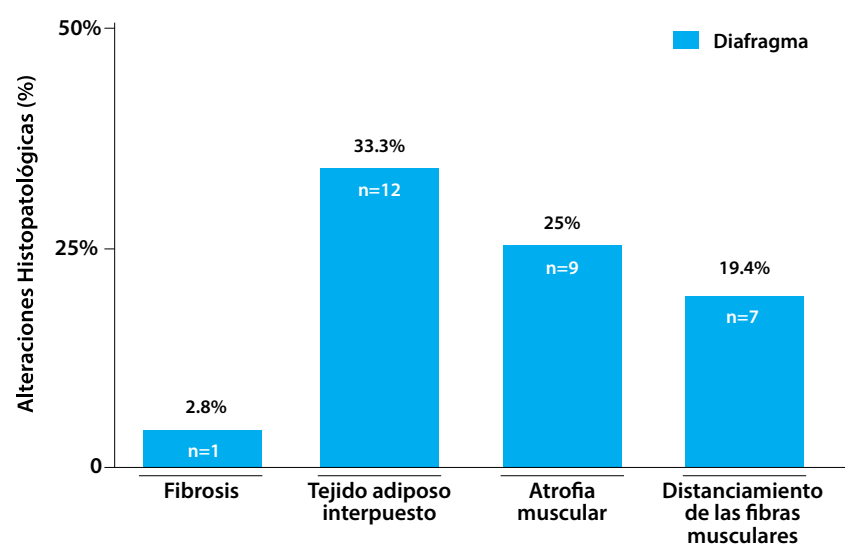

Figura 3. Visualización al microscopio de las biopsias de músculo de diafragma y las alteraciones histopatológicas. Imágenes de cortes histológicos de músculo de diafragma teñidos con la técnica tricrómica de Masson en cadáveres con EPOC: A) Fibrosis muscular (aumento de 40x). Imágenes de láminas histológicas de diafragma teñidas con técnica hematoxilina-eosina (HE) en cadáveres con EPOC: B) Atrofia muscular (aumento 10x). C) Tejido adiposo interpuesto (aumento 20x). D) Fibras separadas (aumento 20x). E) Gráfico representativo de los resultados de las biopsias de músculo de diafragma en cadáveres con EPOC.

Las biopsias de ECOM mostraron resultados que convergieron en el distanciamiento de las fibras (ECOM D: $19,4 \%, n=7 ;$ ECOM I: 16,7\%, $n=6$ ) y la hipertrofia muscular (ECOM D: 13,9\%, n=5; ECOM I: 11,1\%, $n=4$ ), y en menor medida, atrofia muscular (ECOM D y I:
$11,1 \%, \mathrm{n}=4$ ), tejido adiposo interpuesto (ECOM D: $5,6 \%, n=2 ;$ ECOM I: $8,3 \%, n=3$ ) y engrosamiento del tendón (ECOM D y I: 5,6\%, $n=2$ ). Los resultados de las biopsias de ECOM se pueden ver en la Figura 4. A, B, C, D y $E$, respectivamente.
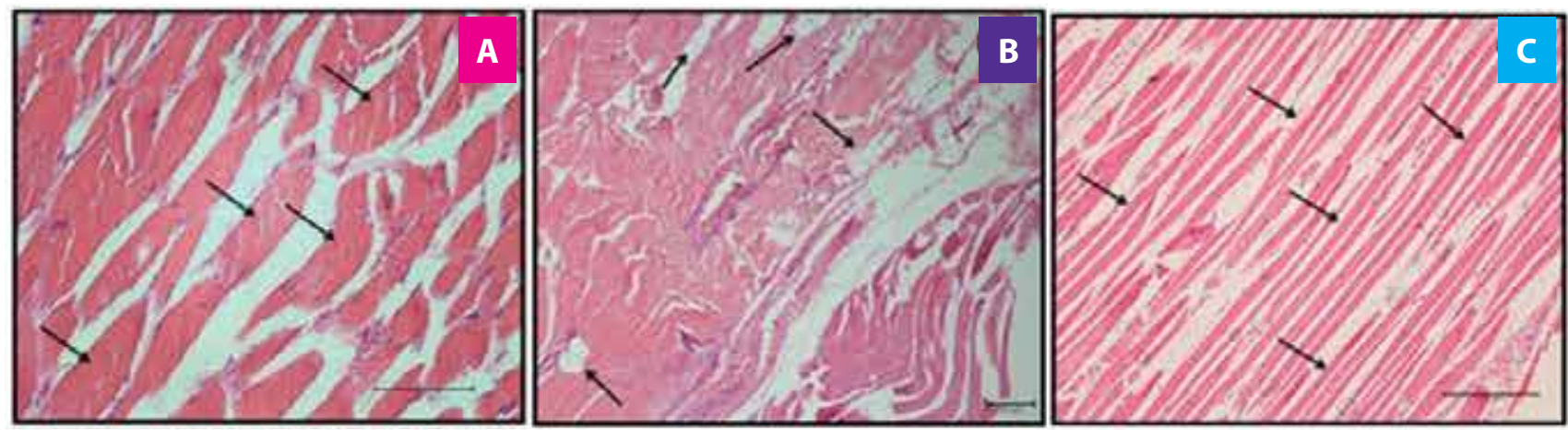

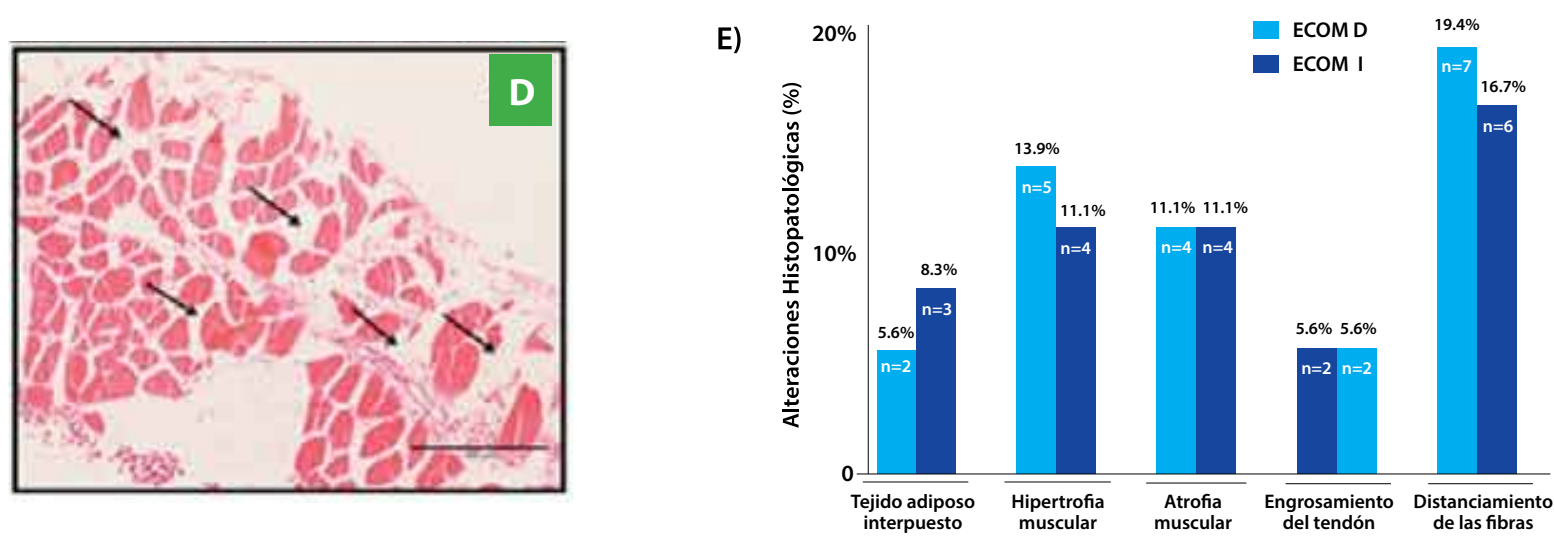

Figura 4. Visualización al microscopio de las biopsias de los músculos esternocleidomastoideos (ECOM) y las alteraciones histopatológicas. Imágenes de cortes histológicos de los músculos esternocleidomastoideos (ECOM) teñidas con la técnica hematoxilina-eosina (HE) en cadáveres con EPOC: A) Hipertrofia de la fibra (aumento 20x). B) Tejido graso interpuesto entre las miofibrillas (aumento 4x). C) Atrofia muscular (aumento 10x). D) Fibras alejadas del ECOM (aumento 10x). E) Gráfico representativo de los resultados de las biopsias entre los músculos esternocleidomastoideos derecho (ECOM D) e izquierdo (ECOM I) en cadáveres con EPOC.

\section{DISCUSIÓN}

La antracosis es una lesión caracterizada por la acumulación de polvo de carbón en los pulmones y, con la consiguiente reacción del tejido a su presencia. De todos los factores exógenos que causan problemas al cuerpo, el más frecuente es el carbón presente como principal contaminante del aire atmosférico. El carbón se inhala como partículas lo suficientemente pequeñas para alcanzar los alvéolos y es fagocitado por macrófagos que pueden devolver su carga de carbón al tejido pulmonar intersticial donde entran a los pulmones. para ser depositados preferentemente en los ganglios linfáticos hiliares y mediastínicos ${ }^{(16)}$.

Por lo tanto, los individuos los fumadores exhiben una pigmentación pulmonar ennegrecida que, cuando se observa debajo de la pleura, está dispuesta en líneas que rodean la periferia de los lóbulos pulmonares. La evidencia de antracosis por contaminación no parece ser un factor predisponente para ninguna enfermedad pulmonar. Sin embargo, cuando se asocia con fumar, agrava los efectos dañinos en los pulmones, este hecho se ha demostrado en estudios realizados con mineros del carbón, y se observó que entre los mineros solo los fumadores desarrollaron alteraciones pulmonares suficientes para producir síntomas ${ }^{(17)}$.

Las ampollas enfisematosas se producen debido a la disminución irreversible en la cantidad de fibras elásticas de los septos alveolares debido a la reducción de la renovación del tejido elástico y su síntesis cualitativamente defectuosa que corresponde a una alteración subpleural o intraparenquimatosa de más de $1 \mathrm{~cm}$ de diámetro y se encuentran rodeados por una pared fibrosa que consiste en pleura visceral, restos de septo alveolar, vasos sanguíneos y antracosis, y su interior está lleno de aire ${ }^{(18)}$. Además, histológicamente, el engrosamiento septal se explica en la mayoría de los casos por un intenso proceso inflamatorio que tiende a cicatrizar, así como por la exacerbación de la reticulina y las fibras de colágeno en los tabiques alveolares ${ }^{(19)}$.

La hiperinflación pulmonar representa uno de los factores críticos capaces de alterar la función de los músculos respiratorios, especialmente cuando induce un acortamiento del diafragma, lo coloca en una posición subóptima, reduce su relación entre la longitud y la tensión y genera su desventaja mecánica, obsrvada en nuestro estudio. Además, las fibras diafragmáticas pueden alterar su disposición mecánica llevando a una capacidad reducida para generar fuerza ${ }^{(11)}$. Por lo tanto, si no hubiera adaptaciones en la longitud del músculo a la hiperinflación crónica, el diafragma permanecería en un estado acortado durante la respiración y su capacidad para contribuir a la presión inspiratoria se reduciría ${ }^{(20)}$.

En el presente estudio, se evidenció que la mayoría de los individuos presentaban cambios estructurales en el diafragma, en los cuales la atrofia muscular era el hallazgo histopatológico más frecuente. La atrofia del diafragma se puede justificar por el aumento de la tasa de proteólisis que se produce en las proteínas musculares dañadas o alteradas. Algunos autores han señalado la posibilidad de que el diafragma y otros músculos respiratorios tengan grados significativamente altos de lesión muscular debido a la apoptosis mionuclear $y / 0$ al estrés oxidativo debido a la sobrecarga ${ }^{(19)}$. 
La degradación de proteínas dañadas o proteínas objetivo que conducen al proceso de atrofia $y$ acortamiento de la fibra muscular se considera uno de los primeros pasos para el proceso de ubiquitinación a través de la proteasoma. Los datos bibliográficos indican que hay un aumento de la actividad de la proteasoma, indicado por la activación de la vía de la ubiquitina-proteasoma, en el diafragma de los pacientes con EPOC ${ }^{(12)}$. Por lo tanto, estos datos sugieren que el daño permanente a las fibras del diafragma puede estar relacionado con sobrecargas inspiratorias y/o cambios en la conformación torácica, lo que contribuye al proceso de remodelación muscular.

La presencia de tejido adiposo interpuesto se encontró en una gran cantidad de biopsias de músculos con diafragma reportado por nuestro estudio lo cual se haya avalado por Conte et al ${ }^{(21)}$ que señalan que el envejecimiento del músculo estriado esquelético es un fenómeno complejo, que consiste en varias alteraciones fisiológicas y morfológicas, como la sustitución de las fibras musculares por tejido adiposo y fibrótico, así como una disminución en la síntesis de proteínas, lo que provoca una reducción de la fuerza y la eficiencia muscular ${ }^{(21)}$. Otro factor predisponente para la interposición del tejido adiposo es la presencia de atrofia muscular que se encuentra en los músculos lumbares multifidiales en pacientes con dolor de pierna evaluado mediante imágenes de resonancia magnética(22).

A partir de esta evidencia, Kader et al. caracterizaron como atrofia muscular leve el reemplazo de menos del $10 \%$ de la masa muscular multifidio con tejido adiposo y fibroso; como atrofia muscular moderada la sustitución entre el $10 \%$ y el $50 \%$; la atrofia severa es el reemplazo de más del $50 \%$ del tejido muscular asociado con la atrofia de otros músculos paravertebrales.

De manera similar, el presente estudio encontró la presencia de atrofia muscular en las biopsias de diafragma, lo que refuerza el hecho de que el tejido muscular puede ser reemplazado por tejido adiposo, confirmado por imágenes histológicas de adipocitos interpuestos entre las miofibrillas. La eliminación de las miofibrillas se puede justificar por la reducción del área de la sección transversal de la fibra diafragmática en la condición de EPOC grave, que puede representar una adaptación estructural beneficiosa, facilitando el transporte de oxígeno y la difusión a través de los capilares en las fibras musculares para satisfacer la demanda metabólica del tejido(12). Otro estudio informó que los niveles de ARN mensajero en el diafragma y el factor de crecimiento vascular endotelial aumentan en los pacientes con EPOC, lo que sugiere una condición de angiogénesis mejorada ${ }^{(23)}$. De esta manera, el desprendimiento de fibras puede ocurrir como un mecanismo adaptativo del tejido muscular en la distribución de nuevos capilares.

La presencia de fibrosis, tejido cicatricial conectivo compuesto por la acumulación de colágeno en el diafragma, fue otro hallazgo interesante. La sobrecarga mecánica puede tener importantes efectos perjudiciales sobre los músculos respiratorios e inducir lesiones ${ }^{(12)}$. Los estudios muestran que los modelos experimentales sometidos a sobrecargas respiratorias altas presentaban lesiones en el diafragma ${ }^{(24,25)}$. A la lesión tisular le sigue la activación de células satélite que proliferan y se diferencian en nuevas células musculares que contribuyen al proceso de reparación con la formación de cadenas pesadas de miosina ${ }^{(25)}$. Sin embargo, algunos estudios han sugerido que la diferenciación de las células satélite del diafragma en nuevas células musculares se ve afectada en la condición de $\operatorname{EPOC}^{(14,20)}$.

Por lo tanto, debido al deterioro del mecanismo de reparación, la sustitución del tejido muscular por el colágeno ocurre como un intento de prevenir lesiones adicionales. Las lesiones y la acumulación de colágeno conducen a la pérdida de la capacidad del individuo con EPOC para generar fuerza. La reducción en el volumen de las fibras musculares en funcionamiento puede ser compensada por hipertrofia o aumento de la activación muscular, sin embargo, debido a que el diafragma está en desventaja mecánica, es incapaz de hipertrofia, promoviendo en algunos casos la atrofia de sus fibras ${ }^{19}$. Un estudio ortopédico ha demostrado que las áreas de sobrecarga muscular pueden llevar a una inflamación del tendón con degeneración y desorganización de las fibrillas de colágeno, una condición asociada con el engrosamiento del tendón ${ }^{(26)}$.

Curiosamente, el diafragma de los pacientes con EPOC moderada y grave se consideró tres veces más susceptible a la ruptura de sus sarcómeros cuando se sometió a ventilación frente a cargas inspiratorias adicionales en comparación con los pacientes sin EPOC ${ }^{(27)}$. Según estos resultados, los datos de un estudio de cadáveres necropsiados con EPOC grave revelaron que un aumento agudo en la sobrecarga respiratoria crónica induce una lesión diafragmática extensa y acumulación de colágeno ${ }^{(19)}$.

Con respecto a las alteraciones tisulares del ECOM 
encontradas en el presente estudio, se sugiere que el efecto de la hiperinflación pulmonar en los músculos inspiratorios, como el intercostal, el escaleno y el ECOM, conduce a un aumento en el diámetro de la caja torácica, lo que limita la eficiencia biomecánica de estos músculos ${ }^{(11)}$. El cambio del tipo de fibra que se produce en direcciones opuestas en el diafragma y otros grupos musculares ha demostrado ser uno de los cambios más frecuentes en los músculos respiratorios de las personas con EPOC. Por lo tanto, mientras que el cambio en el tipo de fibra diafragmática está asociado con la ganancia de fuerza (fibras musculares de tipo I) ${ }^{(28,29)}$.

Es importante destacar que también se observó hipertrofia de ECOM en el presente estudio en un gran número de biopsias. Según Hudson y sus colegas, los pacientes con EPOC necesitan un reclutamiento adicional de los músculos accesorios inspiratorios, especialmente los músculos del ECOM, en un intento por mantener las presiones adecuadas de llenado pulmonar, lo que sugiere que la hipertrofia del ECOM es un intento compensatorio para mejorar su capacidad de ejercicio genera fuerza inspiratoria, ya que el diafragma tenía una capacidad reducida y era más resistente a la fatiga ${ }^{(29)}$.

Curiosamente, algunas biopsias de ECOM mostraron atrofia muscular, lo que corrobora los datos de la literatura que muestran que el envejecimiento favorece la pérdida de motoneuronas $y$, en consecuencia, la denervación de la unión neuromuscular. Estos cambios se producen de manera desigual entre los diferentes grupos musculares, afectando preferentemente a las fibras de tipo II que tienden a estar más presentes en el ECOM y causan cierto grado de atrofia muscular ${ }^{(30)}$.

Se encontraron limitaciones que impidieron un detallar los cambios histopatológicos encontrados en los nervios frénicos en la condición de EPOC. La primera limitación fue la baja adherencia de los familiares a autorizar el formulario del consentimiento informado (ICF) de los cadáveres para la extracción de tejidos para investigación científica. La técnica histológica utilizada en la investigación fue otro obstáculo, ya que no identificó con precisión los cambios patológicos. Por lo tanto, una técnica alternativa para una mejor visualización e identificación de las estructuras en cuestión sería la microscopía electrónica asociada con un software adecuado para mediciones, que ayudaría a profundizar los pesos relacionados con los resultados de este estudio.

\section{CONCLUSIÓN}

Los datos del presente estudio indican que existen cambios histológicos estructurales en el diafragma y en los músculos respiratorios ECOM de los cadáveres afectados por la EPOC, presumiblemente debido a la condición de hiperinflación pulmonar y a los cambios biomecánicos consecuentes en la caja torácica. Sin embargo, se necesitan más estudios para aclarar si tales cambios corresponden a un mecanismo compensatorio para optimizar la ventilación pulmonar o si es una consecuencia de una disfunción sistémica estructural.

Contribuciones de autoría: Los autores participaron en la concepción, redacción y aprobación final del artículo original, además ERQ en la recolección de datos.

Financiamiento: Autofinanciado.

Conflicto de interés: Los autores declaran no tener conflicto de interés en la publicación de este artículo.

Recibido: 30 de octubre del 2019

Aprobado: 13 de diciembre del 2019

\section{Correspondencia: Tales Lyra de Oliveira.}

Dirección: Universidade Municipal de São Caetano do Sul, Faculdade de Medicina, Campus Bela Vista, Rua Treze de Maio, 681, Bela Vista, São Paulo - SP, CEP 01327-000.

Teléfono: $+55112730-0030$

Correo: tales.oliveira@prof.uscs.edu.br

\section{REFERENCIAS BIBLIOGRÁFICAS}

1. Lozano R. et al. Global and regional mortality from 235 causes of death for 20 age groups in 1990 and 2010: a systematic analysis for the Global Burden of Disease Study 2010. Lancet. 2012; 80(9859): 2095-128. doi: 10.1016/ S0140-6736(12)61728-0

2. Mathers $C D$, Loncar D. Projections of global mortality and burden of disease from 2002 to 2030 . PLoS Med. 2006; 3(11):e442. doi: 10.1371/journal. pmed.0030442

3. Dourado VZ. et al. Systemic manifestations in chronic obstructive pulmonary disease. J Bras Pneumol. 2006; 32(2): 161-71. doi: 10.1590/s180637132006000200012

4. Snider GL. Clinical relevance summary: Collagen vs elastin in pathogenesis of emphysema; cellular origin of elastases; bronchiolitis vs emphysema a a cause of airflow obstruction. Chest. 2000; 117(5Suppl 1): 244S-6S. doi 10.1378/chest.117.5 suppl 1.244 s
5. Menezes AM. et al. Chronic obstructive pulmonary disease in five Latin American cities (the PLATINO study): a prevalence study. Lancet. 2005; 366( 9500): 1875-81. doi: 10.1016/S0140-6736(05)67632-5

6. Vogelmeier CF. et al. Global Strategy for the Diagnosis, Management, and Prevention of Chronic Obstructive Lung Disease 2017 Report. GOLD Executive Summary. Am J Respir Crit Care Med. 2017; 195(5): 557-82. doi: 10.1164/rccm.201701-0218PP.

7. Hogg JC, Senior RM. Chronic obstructive pulmonary disease - part 2 pathology and biochemistry of emphysema. Thorax. 2002; 57(9): 830-4. doi: 10.1136/thorax.57.9.830

8. Finkelstein R. et al. Alveolar inflammation and its relation to emphysema in smokers. Am J Respir Crit Care Med. 1995; 152( 5): 1666-72. doi: 10.1164/ ajrccm.152.5.7582312 
9. Stringer K A. et al. Cigarette smoke extract-induced suppression of caspase-3-like activity impairs human neutrophil phagocytosis. Am J Physiol Lung Cell Mol Physiol. 2007; 292(6): 1572-9. doi: 10.1152/ajplung.00325.2006

10. Abboud RT. et al. Relationship of alveolar macrophage plasminogen activator and elastase activities to lung function and CT evidence of emphysema. Chest. 1998; 113(5): 1257-63. Doi: 10.1378/chest.113.5.1257

11. Orozco-Levi M. Structure and function of the respiratory muscles in patients with COPD: impairment or adaptation? Eur Respir J Suppl. 2003; 46: 41-51. doi: 10.1183/09031936.03.00004607

12. Newell SZ, Mckenzie DK, Gandevia SC. Inspiratory and skeletal muscle strength and endurance and diaphragmatic activation in patients with chronic airflow limitation. Thorax. 1989; 44(11): 903-12. doi: 10.1136/ thx.44.11.903

13. Similowski T. et al. Contractile properties of the human diaphragm during chronic hyperinflation. N Engl J Med. 1991; 325(13): 917-23. doi: 10.1056/NEJM199109263251304

14. Ottenheijm CA, Heunks LM, Dekhuijzen R P. Diaphragm adaptations in patients with COPD. Respir Res 2008; 9(12). doi: 10.1186/1465-9921-9-12.

15. Cardoso DM. et al. Effects of expiratory positive airway pressure on the electromyographic activity of accessory inspiratory muscles in COPD patients. J Bras Pneumol. 2011; 37(1): 46-53.

doi: $10.1590 / \mathrm{s} 1806-37132011000100008$

16. Cançado JE. et al. [Clinical repercussions of exposure to atmospheric pollution]. J Bras Pneumol. 2006; 32(2):5-11. doi: 10.1590/s180637132006000800003

17. Churg A. et al. Chronic exposure to high levels of particulate air pollution and small airway remodeling. Environ Health Perspect. 2003; 111(5): 714-8 doi: 10.1289/ehp.6042

18. Gonzalez M. et al. Images in thorax. Tension pneumothorax mimicking giant emphysematous bullae. Thorax. 2010; 65(11): 1028. doi: 10.1136/ thx.2009.129452.

19. Scott A. et al. Increased injury and intramuscular collagen of the diaphragm in COPD: autopsy observations. Eur Respir J. 2006; 27(1): 51-9. doi: $10.1183 / 09031936.06 .00143004$
20. Clanton $T L$, Levine S. Respiratory muscle fiber remodeling in chronic hyperinflation: dysfunction or adaptation? J Appl Physiol (1985). 2009 107(1): 324-35. Doi: 10.1152/japplphysiol.00173.2009

21. Conte M. et al. Differential expression of perilipin 2 and 5 in human skeleta muscle during aging and their association with atrophy-related genes. Biogerontology. 2015; 16(3): 329-40. doi: 10.1152/japplphysiol.00173.2009.

22. Kader DF, Wardlaw D, Smith FW. Correlation between the MRI changes in the lumbar multifidus muscles and leg pain. Clin Radiol. 2000; 55(2): 145-9. doi: 10.1053 /crad.1999.0340

23. Alexopoulou C. et al. Vascular-specific growth factor mRNA levels in the human diaphragm. Respiration. 2005; 72(6):636-41. doi: 10.1159/000089580

24. Zhu E. et al. Diaphragm muscle fiber injury after inspiratory resistive breathing. Am J Respir Crit Care Med. 1997; 155(3): 1110-6.doi: 10.1164/ ajrccm.155.3.9116995

25. CHARGÉ, S. B.; RUDNICKI, M. A. Cellular and molecular regulation of muscle regeneration. Physiol Rev. 2004; 84(1): 209-38. doi: 10.1152/ physrev.00019.2003

26. Factor D, Dale B. Current concepts of rotator cuff tendinopathy. Int J Sports Phys Ther. 2014; 9(2): 274-88. Disponible en: < https://www.ncbi.nlm. nih.gov/pubmed/24790788 >.

27. Whittom F. et al. Histochemical and morphological characteristics of the vastus lateralis muscle in patients with chronic obstructive pulmonary disease. Med Sci Sports Exerc. 1998; 30(10): 1467-74. doi: 10.1097/00005768199810000-00001

28. Satta A. et al. Fibre types in skeletal muscles of chronic obstructive pulmonary disease patients related to respiratory function and exercise tolerance. Eur Respir J. 1997; 10(12): 2853-60. Doi: 10.1183/09031936.97.10122853

29. Hudson AL, Gandevia SC, Butler JE. The effect of lung volume on the coordinated recruitment of scalene and sternomastoid muscles in humans. J Physiol. 2007; 584(1): 261-70. doi: 10.1113/jphysiol.2007.137240

30. Rosenberg IH. Sarcopenia: origins and clinical relevance. Clin Geriatr Med. 2011; 27(3): 337-9. doi: 10.1016/j.cger.2011.03.003

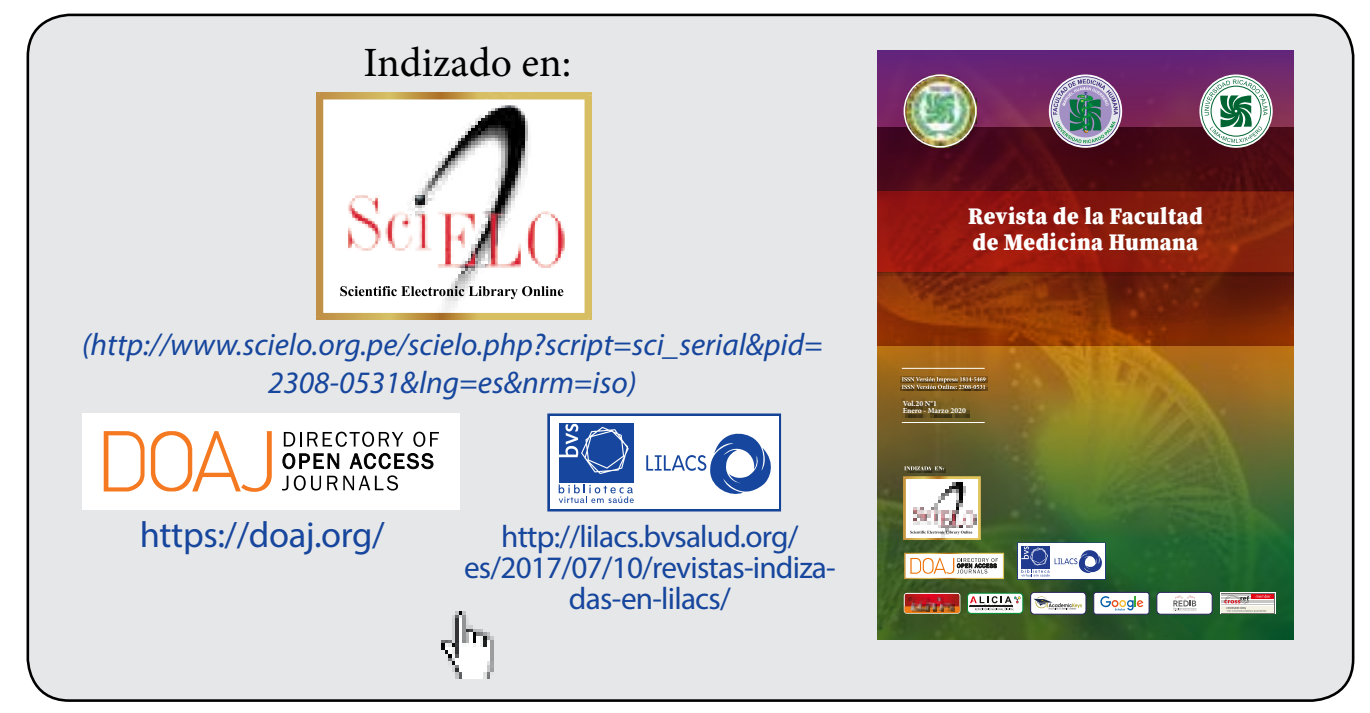

